\title{
A specialized multi-disciplinary care program for children with sepsis and multiple organ dysfunction-associated immune dysregulation
}

\author{
Lindsey R. Swigart ${ }^{1 凶}$, L. Nelson Sanchez-Pinto ${ }^{1}$, Brian E. Nolan ${ }^{2}$, Patrick C. Seed ${ }^{3}$ and Bria M. Coates ${ }^{1}$ \\ (c) The Author(s), under exclusive licence to the International Pediatric Research Foundation, Inc 2022
}

\begin{abstract}
The complex physiology and medical requirements of children with sepsis and multiple organ dysfunction syndrome (MODS) challenge traditional care coordination models. While the involvement of multiple clinical subspecialty services is often necessary to support different care processes and individual organ system dysfunctions, it can also delay the diagnostic process, monitoring, and treatment. The logistics of coordinating with many specialty providers for critically ill patients are challenging and time consuming, and often can result in fragmented communication. To address these and other related issues, we developed a new multi-disciplinary consult service focused on streamlining diagnostics, management, and communication for patients with sepsis and MODS-associated immune dysregulation. The service, called the Program in Inflammation, Immunity, and the Microbiome (PrllMe), is now a hospital-wide clinical consult service at our institution caring for a broad group of patients with immune dysregulation, particularly focusing on patients with sepsis and MODS. In this paper, we summarize the development, structure, and function of the program, as well as the initial impact. This information may be helpful to clinicians and healthcare leaders who are developing multi-disciplinary consult services for children with complex care needs, especially those with sepsis and MODS-associated immune dysregulation.
\end{abstract}

Pediatric Research (2022) 91:464-469; https://doi.org/10.1038/s41390-021-01891-y

\section{IMPACT:}

- The care of children with sepsis and multiple organ dysfunction-associated immune dysregulation requires rapid and flexible involvement of multiple clinical subspecialists that is difficult to achieve without fragmented care and delayed decision making.

- In this narrative review we describe the development, structure, and function of a multi-disciplinary consult service at a children's hospital dedicated to helping coordinate management and provide continuity of care for patients with sepsis and multiple organ dysfunction-associated immune dysregulation.

- This information may be helpful to clinicians and healthcare leaders who are developing multi-disciplinary consult services for children with complex care needs, especially those with sepsis and MODS-associated immune dysregulation.

\section{INTRODUCTION}

The majority of patients with pediatric sepsis have favorable outcomes, with mortality rates of less than $10 \%{ }^{1}$. However, when sepsis leads to multiple organ dysfunction syndrome (MODS), the risk of mortality increases in proportion to the number of organ systems affected ${ }^{2}$. Immune dysregulation is a hallmark of pediatric severe sepsis and MODS, leading to prolonged hospitalization and increased late mortality ${ }^{3-5}$. Carcillo et al. ${ }^{3}$ describe three phenotypes of immune dysregulation in sepsis, including sequential organ failure, thrombocytopenia-associated multiple organ failure (TAMOF) due to an immune-mediated thrombotic microangiopathy, and a state of acquired immune deficiency sometimes termed sepsis-associated immune paralysis $(\mathrm{SAI})^{3}$. Additionally, hyperinflammatory sepsis with secondary macrophage activation syndrome (MAS) has been described by Carcillo and others ${ }^{6-8}$. The mechanisms behind these phenotypes of immune dysfunction are complex and may depend on the child's genetic predisposition, overall immune phenotype, and clinical course ${ }^{5}$.

Children with long-term sequelae of sepsis and MODS are not only physiologically and medically complex but coordinating their care can also become a challenging task for the primary medical team. Maintaining strong lines of communication among multiple subspecialty services is required and contributes to the challenge of caring for these patients. Often, these patients require intensive care unit (ICU)-level care along with consultation by many subspecialty services to address individual organ system dysfunctions. In traditional models of care for these children, the engagement of subspecialty consultation services, synthesis of multiple streams of information, coordination of care, and, ultimately, the development of a care plan, often falls to the primary clinical team ${ }^{9}$. Unfortunately, this care model often results in conflicting and asynchronous recommendations that

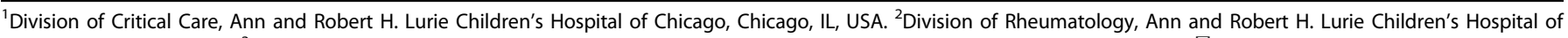
Chicago, Chicago, IL, USA. ${ }^{3}$ Division of Infectious Diseases, Ann and Robert H. Lurie Children's Hospital of Chicago, Chicago, IL, USA. ${ }^{凶}$ email: Iswigart@luriechildrens.org

Received: 17 June 2021 Revised: 10 September 2021 Accepted: 29 November 2021

Published online: 12 January 2022 
Table 1. Patients with sepsis and MODS in our pediatric ICU between 2015 and 2018.

\begin{tabular}{|c|c|c|c|c|}
\hline Criteria & $\begin{array}{l}\text { Day } 1 \\
n(\%)\end{array}$ & $\begin{array}{l}\text { Died }^{a} \\
n(\%)\end{array}$ & $\begin{array}{l}\text { Progressive MODS } \\
n(\%)\end{array}$ & $\begin{array}{l}\text { Persistent MODS } \\
n(\%)\end{array}$ \\
\hline All MODS ${ }^{d}$ & 1627 & $94(5.8)$ & $655(40.3)$ & $324(19.9)$ \\
\hline MODS with hypoxia, shock ${ }^{f}$ & 331 & $61(18.4)$ & 185 (55.9) & $111(33.5)$ \\
\hline Sepsis-associated MODS & 779 & $70(9.0)$ & $409(52.5)$ & $254(32.6)$ \\
\hline
\end{tabular}

an-hospital death.

${ }^{\mathrm{b}}$ Additional organ dysfunctions or death between day 2 and 7 of admission.

'Persitent MODS at 7 days from admission or death within the first week.

${ }^{\mathrm{d}}$ Multiple organ dysfunction syndrome (MODS): 2 or more organ dysfunctions on day 1 of admission.

${ }^{\text {e }}$ Respiratory dysfunction based on oxygenation index.

${ }^{f}$ Cardiovascular dysfunction-based vasoactive support.

lag behind the dynamic progression of the patient's illness. When multiple subspecialties contribute to the care of these patients in an uncoordinated way, a shared mental model is often lacking, which can result in broken communication and fragmented care $^{10-12}$. Additionally, in the traditional model of rotating providers, particularly in the ICU and other inpatient services, there can be a lack of continuity during the extended hospital stays common in these patients with complex needs. This can make keeping track of specialized diagnostic test results and long-term treatment plans more challenging.

Multi-disciplinary teams where individual members have a sense of accountability, a shared mental model, and common goals can enhance patient care and provide effective team management processes that are safer for patients ${ }^{13}$. Multidisciplinary cooperation for rare and complex pediatric disorders has previously been implemented with success in outpatient care areas, including for various genetic disorders ${ }^{14-16}$ and vascular malformations ${ }^{17}$. Like other large pediatric care centers, our institution supports a number of multi-disciplinary services in the outpatient setting, including a vascular lesion clinic, a muscular dystrophy clinic, a cleft lip and palate clinic, and others that convene and provide care primarily in the outpatient setting. The multi-disciplinary approach to immunologically complex patients has also been recognized as a need in academic children's hospitals. These patients, however, frequently require multidisciplinary care in a continuum between the inpatient and outpatient settings. One of few examples of this type of program is the immune dysregulation team at the Children's Hospital of Philadelphia that provides both inpatient and outpatient services (private communication). We recognized that despite representing a small proportion of the overall population of patients admitted to our hospital, patients with sepsis and MODSassociated immune dysregulation are among those at highest risk of morbidity and mortality (Table 1). To address the challenges of a team-based approach to the care of these patients, we developed a dedicated multi-disciplinary consult service for children with sepsis and MODS-associated immune dysregulation at our tertiary urban children's hospital in Chicago, IL. Our program, now called the Program in Inflammation, Immunity, and the Microbiome (PrllMe), began development in 2019 and resulted in the launch of our clinical consult Critical Inflammation Team, commonly referred to as the PrllMe team, in January 2020. We hypothesized that a more standardized care process with a core multi-disciplinary group of providers would shorten the time to diagnosis, individualize therapy, and provide continuity to improve outcomes.

In this narrative review, we will describe the development, structure, and daily operations of our program, and discuss the role of care coordination and communication in our multidisciplinary consult team. This information may be helpful to clinicians and healthcare leaders who are developing multidisciplinary consult services for children with complex care needs, especially those with sepsis and MODS-associated immune dysregulation.

\section{PROGRAM DEVELOPMENT}

In early 2019 we completed a structured gap analysis to direct key stakeholders to consider improvement areas, including those of personnel, technology, and processes for the management of children with sepsis and MODS-associated immune dysregulation. The broad goals of the team were formulated through several brainstorming sessions with representation of key clinical and leadership stakeholders in the hospital, including representatives from critical care, infectious diseases, rheumatology, immunology, gastroenterology, hepatology, hematology, oncology, pediatric surgery, and transplant surgery, among others.

The goals identified for the program were to:

1. Coordinate the diagnostic process and synergize emergent diagnostic testing, therapeutics, monitoring, and supportive care that may be fragmented and inefficient.

2. Serve as a nexus for advanced diagnostics, including immuno-phenotyping.

3. Provide patients and families with streamlined access to cutting-edge therapies.

4. Act as a translational research and innovation hub.

Once these goals for the PrllMe team were established, we began to work on a dedicated program development plan. A pediatric critical care advanced practice nurse (APN) was hired as the clinical coordinator and continuity provider for the team. A group of subspecialists were identified based on necessity, interest, and availability to serve as the core attending physicians on the team, including members from critical care, immunology, rheumatology, infectious disease, hematology, oncology, gastroenterology, and hepatology. A rheumatologist with a specific interest in immune dysregulation syndromes was also recruited with dedicated clinical time for PrllMe. Once the team members were identified, an outside business consulting group was engaged to collaborate on a process and structure for day-today operations. The design process was refined through a set of focused interviews with relevant stakeholders and hospital departments, as well as a one-day team development session using Design Thinking principles ${ }^{18}$. Goals for communication strategies and important team functions were identified, and a process for a multi-disciplinary consult with supporting documentation in the electronic health record were developed. We shared our proposed patient population and process with key provider groups across the hospital. Ultimately, we launched the 
Critical Inflammation Team, the patient-facing clinical consult service, in January 2020.

\section{DAILY OPERATIONS OF THE PROGRAM \\ Staffing}

Historical data from our institution (Table 1) suggested we could expect 1-3 consults per week. We used these numbers to inform team staffing. The team is staffed Monday through Friday during daytime hours by a dedicated APN and an attending physician who rotates weekly from a core group of subspecialty services, including infectious diseases, immunology, rheumatology, and critical care. Typically, the attending physician covers both their subspecialty inpatient team while also serving as the PrllMe attending. The team has additional core members from oncology, hematology, hepatology, neuro-critical care, and gastroenterology who participate in consults but do not rotate on service. We have closely partnered with our pathology lab, immunology lab, and genetic counselors to provide additional diagnostic recommendations. The APN also serves as the clinical coordinator for the team in an administrative support role, organizing monthly patient case reviews, administrative team meetings, maintaining a patient registry, and performing process improvement.

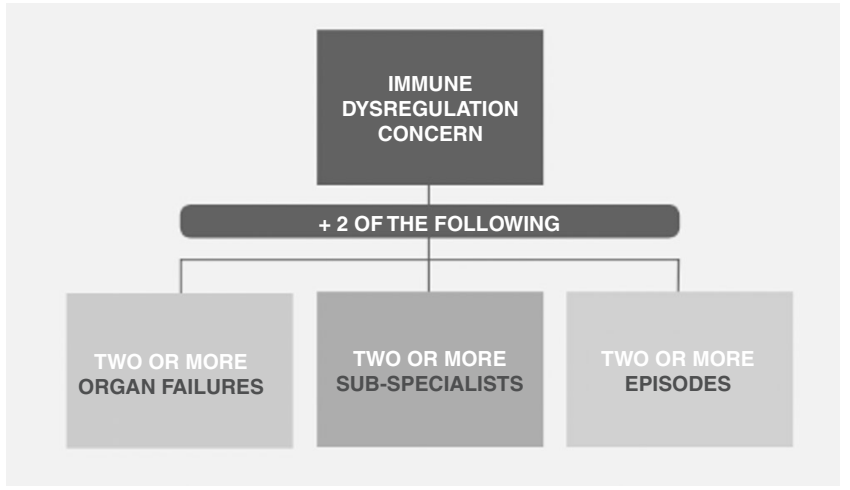

Fig. 1 PrIIMe consult criteria. General patient characteristics to guide consideration for PrllMe consult.

\section{Consult criteria}

In order to capture the patient population we intended to target, we developed a set of consult criteria that we shared hospitalwide as part of our clinical service launch. Our broad goal is to capture patients with suspicion for immune dysregulation and multiple organ involvement. Specifically, our criteria are: patients with any concern for immune dysregulation where $\geq 2$ organ systems are involved, $\geq 2$ subspecialty teams are consulting, and/ or there is a cyclic or episodic nature to the patient's illness (Fig. 1). The primary team consults the PrlIMe APN with the clinical question related to immune dysregulation. Most often these consult questions are related to diagnostic difficulty in patients with known or suspected immune dysregulation syndromes (primary or secondary), or workup and management of critical MODS where there has been a lack of response to traditional therapies.

\section{Consult process}

Our consult includes extensive history review related to the clinical question and facilitation of a care discussion with all relevant providers to create a multi-disciplinary diagnostic and management plan. Figure 2 provides a visual example of a PrlIMe consult process for a critically ill patient. Once the primary team engages the PrlIMe team, the APN begins initial chart review and history-taking with the patient focusing on their immune phenotype including infection history, inflammatory marker and lab trends, past functional immune testing, past genetic testing, organ dysfunctions, and history of symptoms. This is used to inform an initial decision between the PrlIMe APN and the PrllMe attending as to which subspecialties are needed to facilitate an informative discussion of the patient's disease process. Then a care discussion is organized with as many relevant providers as able. This process is illustrated in Fig. 3. Consults most often are completed within the same day, although our team is flexible to include more rapid consultation and recommendations for unstable critically ill patients, as well as longer turnaround times when extensive review of a chronic issue is required. We use an institutionally approved, secure virtual platform (Microsoft ${ }^{\circledR}$ Teams, Microsoft Corporation, Redmond, WA) for our consult discussion to make it possible for providers to join from outside of clinical areas and may include

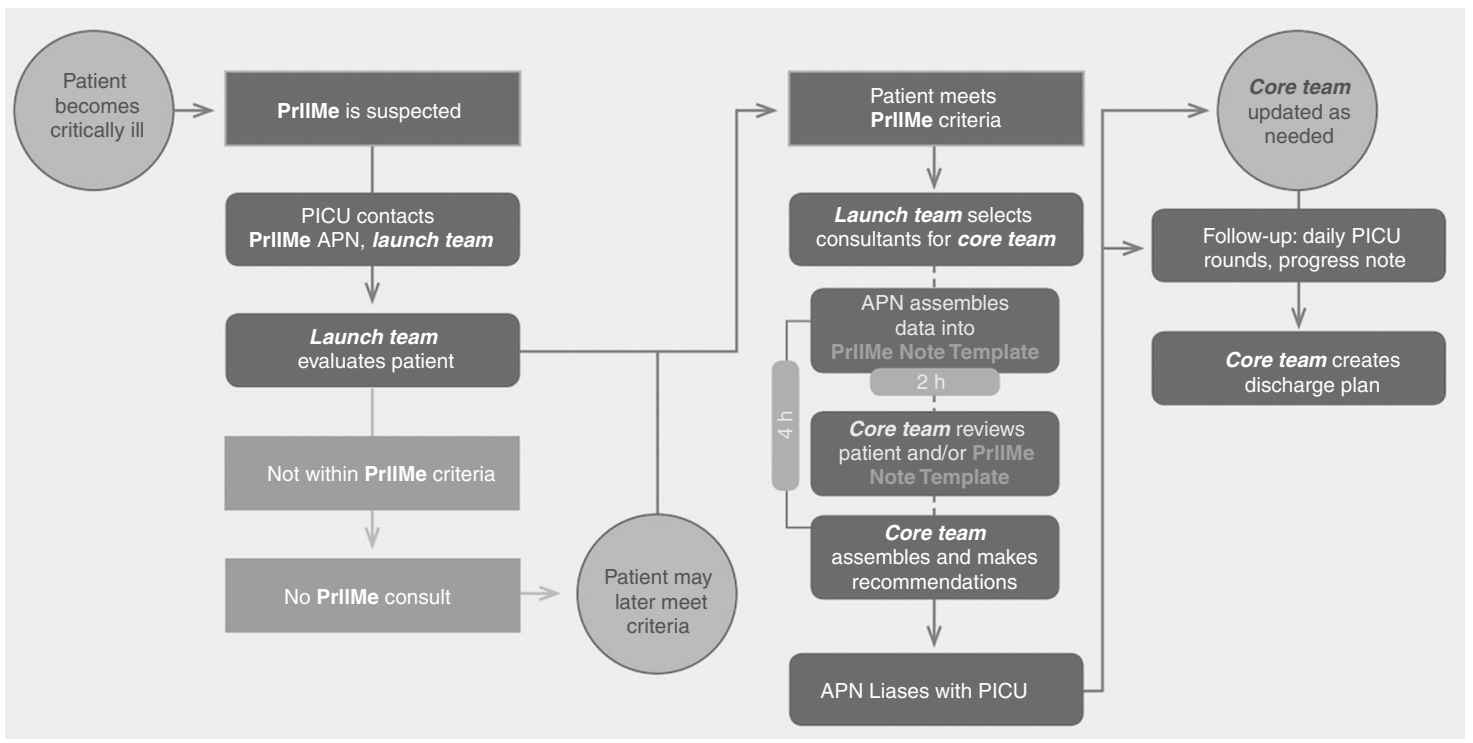

Fig. 2 PrIIMe Team Process. This figure is a flow chart that depicts the key components of a PrllMe consult for a critically ill patient. Launch team refers to the patient's primary service providers and the on-service PrIIMe APN and attending. Core team refers to the broad PrlIMe team including all relevant specialists and additional PrllMe team members participating in the initial consult discussion and any follow-up as needed. 




Fig. 3 PrIIMe Team Structure. This figure depicts the key providers involved in a PrllMe consult in a critically ill patient, including the primary ICU team who requests the consult and conveys the consult needs, the on-service PrIIMe team (APN and attending), the additional core PrIIMe team members who may participate in the consult, and the additional specialists as needed customized to the patient's clinical status.

Table 2. Clinical characteristics of patients receiving PrllMe consults.

\begin{tabular}{|c|c|}
\hline Clinical variables & Patients, $\boldsymbol{n}(\%)$ \\
\hline \multicolumn{2}{|l|}{ Location } \\
\hline Pediatric ICU & $39(50)$ \\
\hline Cardiac ICU & $14(18)$ \\
\hline Neonatal ICU & $1(1.3)$ \\
\hline Inpatient floor & $22(28)$ \\
\hline Outpatient & $2(2.7)$ \\
\hline Mechanically ventilated & $26(33)$ \\
\hline Vasoactive support & $31(40)$ \\
\hline \multicolumn{2}{|l|}{ Consult type $^{a}$} \\
\hline Critical inflammation $^{\mathrm{b}}$ & $38(48.7)$ \\
\hline Diagnostic difficulty & $30(38.5)$ \\
\hline Chronic critical illness & $3(3.8)$ \\
\hline Sepsis-associated immune paralysis & $13(16.6)$ \\
\hline$>1$ reason for consult & $9(13)$ \\
\hline \multicolumn{2}{|l|}{ Disposition } \\
\hline Home & $42(54)$ \\
\hline Home with outpatient rehabilitation & $6(9)$ \\
\hline Inpatient rehabilitation & $5(7)$ \\
\hline Long-term care facility & $1(1)$ \\
\hline Death & $18(23)$ \\
\hline Remains inpatient & 6 (6) \\
\hline
\end{tabular}

those outside the institution, if needed, for additional insight. The PrllMe team members who are not on clinical service during these consult discussions often provide a fresh perspective on the patient's case and help the team consider a broader differential and potentially new management strategies. Timing of consult discussions is balanced to ensure that key stakeholders are available to participate. PrllMe providers are dedicated to participating in these consults. Providers most often participating as part of PrllMe include infectious disease (87.5\% of consults), rheumatology (65.3\% of consults), immunology $(43.1 \%$ of consults), critical care (37.5\% of consults), and hematology/oncology (29.2\% of consults). Neurology, kidney, genetics, and pathology input is common (27.5\% of consults). Between 5 and 15 providers typically join consult discussions, depending on the patient's needs. Challenges to consult timing include providers' other clinical duties (clinic appointments, inpatient service needs) and laboratory or research duties. If questions arise for providers who are not able to attend the consult discussion, the APN will follow-up with dedicated questions. Once the group recommendations are finalized, the APN summarizes them into a consult note in the electronic medical record. This note was specifically developed for the PrllMe team with our institution's billing department and health information management department to allow for multidisciplinary documentation. Key subspecialists contributing to the management of the patient addend the note with their specific recommendations and clarifications. This reduces the overall burden of documentation on complex patients and allows for all issues to be documented in a single note. Finally, to ensure broad team awareness of all PrllMe patients, the APN sends a weekly signout to all PrlIMe team members.

\section{OUTCOMES}

Since our launch, while our team was consulted on average for one patient per week, our activities were considerably affected by the reduced overall patient volumes our hospital saw due to the COVID-19 pandemic. In total, we have cared for 78 patients with varying forms of immune dysregulation, initially only in the ICUs but now expanded to all inpatient units. Table 2 summarizes the clinical characteristics of our patients, as tracked in our patient registry. Our heterogeneous population can be divided into four major categories: $48.7 \%$ critical inflammation $(n=38)$, $38.5 \%$ diagnostic difficulty with suspected immune dysregulation $(n=30), 16.6 \%$ sepsis-associated immune paralysis $(n=13)$, and $3.8 \%$ chronic critical illness with known or suspected immune dysregulation $(n=3)$. We are consulted within a wide range of time during the inpatient stay, with the majority of consults occurring within the first day to week of hospitalization, though 12 consults were $>1$ month into hospital stay, and 2 consults were $>1$ year into hospitalization. Our patients' mean length of stay is 23 days ( $\min 1, \max 390$ ).

The variety of expertise on our team allows us to focus on the overlap between critical care and immunology, host responses to pathogens, and resulting variable end-organ dysfunctions. We developed standardized consult plans for common consult types 
including SAl, hyperinflammation syndromes such as MAS, TAMOF, and severe COVID-related multi-system inflammatory syndrome in children (MIS-C). However, the population is very heterogeneous and often requires a patient-specific, personalized approach. We partner with our immunology lab to offer novel cellbased testing and biomarker evaluation specific to immune paralysis and other forms of immune dysregulation, including assessment of monocyte HLA-DR expression as a biomarker for SAI not previously used at our institution. We also partner closely with our genetics team to consider genetic testing beyond the whole exome to include intronic or structural mutations that are rare but significant, as well as whole-genome testing and interpretation. We have identified several probable causative mutations in our patients, including a newly described CHRNA3 mutation $^{19}$, and two structural RAB27a mutations leading to diagnosis of atypical Griscelli Syndrome ${ }^{20}$. Additionally, with the COVID-19 pandemic erupting shortly after our clinical launch, we leveraged our multi-disciplinary approach to care for several cases of refractory MIS-C and provide clinical best practice recommendations as the evidence emerged.

\section{PATIENT FOLLOW-UP}

In-hospital care from our team varies depending on patient and provider needs. Sometimes recommendations are summarized in a one-time consult with a specific clinical question and targeted recommendations. Other patients benefit from the development of a long-term care relationship, especially those with recurrent or more complex conditions. The APN and the on-service attending round on established patients while inpatient. If major clinical changes occur with the patient after the initial care discussion, we often reconvene the multi-disciplinary group to discuss further recommendations. If subsequent admissions occur, the PrllMe team may again be engaged. As such, we aim to create a collective knowledge base and institutional memory by following patients with sequelae of immune dysregulation that currently lack a medical home in another pediatric subspecialty service.

Until we are able to develop a PrllMe-specific outpatient clinic, the current practice of our service is to utilize our inpatient diagnostic plan to better identify the patient's immune dysregulation and phenotype and then an outpatient provider who may serve as a consultant of reference for the patient's care related to immune dysregulation. This is often a member of our team from immunology or rheumatology who has become familiar with the patient's course through the PrllMe consult and is well-versed in caring for immune dysregulation syndromes. This facilitates outpatient coordination and continuity that may have been lacking prior to the inpatient stay.

\section{PROGRAM EVALUATION}

To emphasize continued process improvement, we have a monthly patient review for discussion of the previous month's consults including details of their care, what went well, and what could be improved, so we can we implement changes in real time as needed. We participate actively in morbidity and mortality reviews involving our patients.

We send a follow-up survey to the providers who consult the PrllMe team on their patients. This anonymous survey created in REDCap is sent via email to all providers who participated in a patient's initial PrllMe consult discussion, including residents $(n=$ $14,31.1 \%)$, fellows $(n=8,17.6 \%)$, advanced practice providers ( $n=11,23.4 \%)$, hospitalists $(n=4,8.9 \%)$, and attending physicians $(n=10,22.2 \%)$. Currently the survey has a $34.7 \%$ response rate (48/138). Likert Scales are used to assess providers' rating of how PrllMe impacted patient care via diagnostic and treatment recommendations, documentation, safety of care, and clarity of communication. Moderate to significant changes in diagnostics and treatment were both perceived $71.7 \%$ of the time $(n=33)$, and $66 \%(n=31)$ of respondents "completely agree" that PrllMe added to the overall care of their patient. Communication between providers and PrllMe was rated as "good" or "excellent" in all responses ( $n=45,3$ missing data). Almost $100 \%$ of providers would consult PrlIMe again ( $n=46,1$ missing data). Open ended comments are also available, and show a positive reception of our team by other providers including comments like: "Expertise in many areas that come together to think differently about our patients"; "Excellent communication and documentation. This service is so helpful for a specific type of diagnostic dilemma where you really need multidisciplinary input", and "Help in an area that is very specialized and very much needed".

\section{CONCLUSION}

Multi-disciplinary care teams have been created for a variety of pediatric syndromes to address complex care needs in a safe and streamlined way. We noticed a gap in care for critically ill patients with sepsis and multiple organ system involvement, specifically those with various forms of immune dysregulation. We launched the PrllMe team to address the needs of these vulnerable patients with the aim to eventually improve outcomes by creating a standard care process for multi-disciplinary communication, diagnostics, and development of individualized treatment plans. Our consult service has now successfully launched and grown over its first year, with continued plans for expansion of our clinical and research efforts.

\section{REFERENCES}

1. Weiss, S. L. et al. The epidemiology of hospital death following pediatric severe sepsis: when, why, and how children with sepsis die. Pediatr. Crit. Care Med. 18, 823-830 (2017).

2. Leteurtre, S. et al. Validation of the paediatric logistic organ dysfunction (PELOD) score: prospective, observational, muticentre study. Lancet 362, 129-197 (2003).

3. Carcillo, J. A. et al. A multicenter network assessment of three inflammation phenotypes in pediatric sepsis-induced multiple organ failure. Pediatr. Crit. Care Med. 20, 1137-1146 (2019).

4. Hall, M. W. et al. Immunoparalysis and nosocomial infection in children with multiple organ dysfunction syndrome. Intensive Care Med. 37, 525-532 (2011).

5. Carcillo, J. A. et al. Pathophysiology of pediatric multiple organ dysfunction syndrome. Pediatr. Crit. Care Med. 18, S32-S45 (2017).

6. Karakike, E. \& Giamarellos-Bourboulis, E. J. Macrophage activation-like syndrome: a distinct entity leading to early death in sepsis. Front. Immunol. 10, 55 (2019).

7. Kuwata, K., Yamada, S., Kinuwaki, E., Naito, M. \& Mitsuya, H. Peripheral hemophagocytosis: an early indicator of advanced systemic inflammatory response syndrome/hemophagocytic syndrome. Shock 25, 344-350 (2006).

8. Carcillo, J. A. et al. Three hypothetical inflammation pathobiology phenotypes and pediatric sepsis-induced multiple organ failure outcome. Pediatr. Crit. Care Med. 18, 513-523 (2017)

9. Miller, A., Weinger, M. B., Buerhaus, P. \& Dietrich, M. S. Care coordination in intensive care units: communicating across information spaces. Hum. Factors $\mathbf{5 2}$, 147-161 (2010).

10. Alvarez, G. \& Coiera, E. Interdisciplinary communication: an uncharted source of medical error? J. Crit. Care 21, 236-242 (2006).

11. Adams, $\mathrm{S}$. et al. Perspectives on team communication challenges in caring for children with medical complexity. BMC Health Serv. Res. 21, 1-14 (2021).

12. Kuo, D. Z., McAllister, J. W., Rossignol, L., Turchi, R. M. \& Stille, C. J. Care coordination for children with medical complexity: whose care is it, anyway? Pediatrics 141, S224-S232 (2018).

13. Stocker, M., Pilgrim, S. B., Burmester, M., Allen, M. L. \& Gijselaers, W. H. Interprofessional team management in pedatric critical care: some challenges and possible solution. J. Multidiscip. Healthc. 9, 47-58 (2016).

14. Grosse, S. D. et al. Models of comprehensive multidisciplinary care for individuals in the United States with genetic disorders. Pediatrics 123, 407-412 (2009).

15. Kodolitsch, Y. V. et al. The role of the multidisciplinary health care team in the management of patients with Marfan Syndrome. J. Multidiscip. Healthc. 3, 587-614 (2016).

16. Auvin, S. et al. A step-wise approach for establishing a multidisciplinary team for the management of tuberous sclerosis complex: a Delphi consensus report. Orphanet J. Rare Dis. 14, 1-10 (2019). 
17. Rochon, P. J. Importance of multidisciplinary approach to vascular malformation management. Semin. Intervent. Radiol. 34, 301-302 (2017).

18. Brown, T. Design thinking. Harv. Bus. Rev. 86, 84 (2008).

19. Shibao, C. A. et al. Familial autonomic ganglionopathy caused by rare CHRNA3 genetic variants. Am. Acad. Neurol. 97, e145-e155 (2021).

20. Cianca Tesi, M. et al. A RAB27A 59 untranslated region structural variant associated with late-onset hemophagocytic lymphohistiocytosis and normal pigmentation. J. Allergy Clin. Immunol. 142, 317-321 (2018).

\section{ACKNOWLEDGEMENTS}

The authors would like to acknowledge the support of the entire PrllMe team in the creation and success of this program, including Aisha Ahmed, Amer Khojah, Joanna Weinstein, Leena Mithal, Taylor Heald-Sargent, Allison Remiker, Ravi Jhaveri, Ami Patel, Mehreen Arshad, and Jeffery Brown. We would also like to acknowledge the Lurie Children's Immunology Lab including Aaruni Khanolkhar and Nicolas Benson, and the Lurie Children's Genetics Division, including Kai Lee Yap and Alexander Ing for supporting the diagnostics arm of the team. We would like to thank all the patients and families cared for by the PrllMe team for allowing us to be a part of their medical experience and teaching us every day.

\section{AUTHOR CONTRIBUTIONS}

All authors made substantial contributions to the conception of this paper. L. Nelson Sanchez-Pinto provided data and analysis. All authors contributed to drafting and revising the content. All authors provided final approval of this version to be published.

\section{COMPETING INTERESTS}

The authors declare no competing interests.

\section{ADDITIONAL INFORMATION}

Correspondence and requests for materials should be addressed to Lindsey $R$. Swigart.

Reprints and permission information is available at http://www.nature.com/ reprints

Publisher's note Springer Nature remains neutral with regard to jurisdictional claims in published maps and institutional affiliations. 